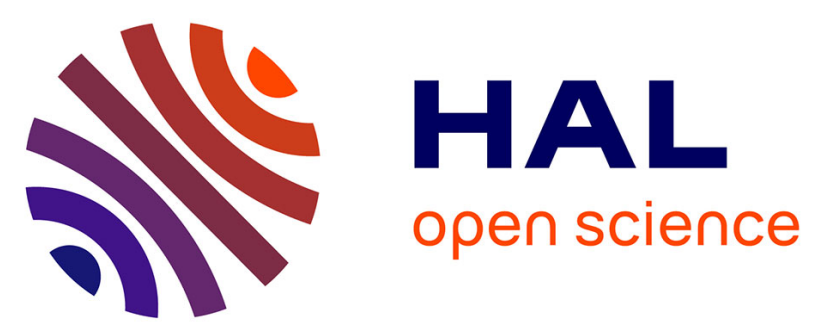

\title{
Hydrogenated graphene on $\operatorname{Ir}(111)$ : A high-resolution electron energy loss spectroscopy study of the vibrational spectrum
}

Line Kyhl, Richard Balog, Thierry Angot, Liv Hornekaer, Régis Bisson

\section{- To cite this version:}

Line Kyhl, Richard Balog, Thierry Angot, Liv Hornekaer, Régis Bisson. Hydrogenated graphene on $\operatorname{Ir}(111)$ : A high-resolution electron energy loss spectroscopy study of the vibrational spectrum. Physical Review B: Condensed Matter and Materials Physics (1998-2015), 2016, 93, pp.115403. 10.1103/PhysRevB.93.115403 . hal-01284577

\section{HAL Id: hal-01284577 \\ https://hal-amu.archives-ouvertes.fr/hal-01284577}

Submitted on 7 Mar 2016

HAL is a multi-disciplinary open access archive for the deposit and dissemination of scientific research documents, whether they are published or not. The documents may come from teaching and research institutions in France or abroad, or from public or private research centers.
L'archive ouverte pluridisciplinaire HAL, est destinée au dépôt et à la diffusion de documents scientifiques de niveau recherche, publiés ou non, émanant des établissements d'enseignement et de recherche français ou étrangers, des laboratoires publics ou privés. 


\title{
Hydrogenated graphene on $\operatorname{Ir}(111)$ : A high-resolution electron energy loss spectroscopy study of the vibrational spectrum
}

\author{
Line Kyhl, ${ }^{1, *}$ Richard Balog, ${ }^{2}$ Thierry Angot,${ }^{3}$ Liv Hornekær,${ }^{1,2, \dagger}$ and Régis Bisson ${ }^{3, \ddagger}$ \\ ${ }^{1}$ iNANO, University of Aarhus, DK-8000 Aarhus $C$, Denmark \\ ${ }^{2}$ Institute of Physics and Astronomy, University of Aarhus, DK-8000 Aarhus C, Denmark \\ ${ }^{3}$ Aix-Marseille Université, CNRS, PIIM UMR 7345, 13397 Marseille, France
}

(Received 20 November 2015; revised manuscript received 18 January 2016; published 4 March 2016)

\begin{abstract}
Hydrogen atom adsorption on high-quality graphene on $\operatorname{Ir}(111)[\operatorname{gr} / \operatorname{Ir}(111)]$ is investigated using highresolution electron energy loss spectroscopy. The evolution of the vibrational spectrum, up to $400 \mathrm{meV}$, of $\mathrm{gr} / \operatorname{Ir}(111)$ upon increasing hydrogen atom exposures is measured. The two dominant binding configurations of atomic hydrogen are identified as (1) graphanelike hydrogen clusters on the parts of the graphene more strongly interacting with the $\operatorname{Ir}(111)$ surface and (2) dimers bound more weakly to the freestanding parts of the graphene. The graphanelike surface structures lead to increased corrugation of the graphene sheet, yielding graphane-related phonon components. Additionally, a recent theoretical prediction of the existence of a bending character for a LO/TO graphane chair phonon mode is experimentally verified. No clear evidence was found for hydrogen bound on both sides of a high-quality graphene sheet and phonon features strongly suggest interactions between graphanelike hydrogen clusters and Ir atoms in the substrate.
\end{abstract}

DOI: 10.1103/PhysRevB.93.115403

\section{INTRODUCTION}

The isolation and study of graphene, a single layer of carbon atoms in an $s p^{2}$ honeycomb configuration, was first presented in 2004 [1]. Graphene has since attracted increased attention, initially due to its outstanding and unusual electronic properties [1-4], exhibiting carrier mobilities of $\sim 200000 \mathrm{~cm}^{2} \mathrm{~V}^{-1} \mathrm{~s}^{-1}$ [5], and later due to its potential application in areas such as photonics [6,7], corrosion protection [8,9], antibiotics [10], filtration [11] and mechanically enhanced composite materials [12]. It has been shown that the properties of a graphene sheet, such as the mechanical $[13,14]$, optical [15,16], and electronic [17-20] properties, can be altered by chemical functionalization. This ability to control the properties of graphene is crucial for realizing its application in industrial uses. The adsorption mechanism, saturation level, and configuration of any chemisorbed species are highly influenced by the level of interaction of the graphene with the underlying substrate [21-23]. Hydrogen adsorption provides a well-studied example of chemical functionalization of graphene on a number of substrates [21,22,24,25]. On graphite and quasifreestanding graphene on hydrogen intercalated $\mathrm{SiC}$ (gr/H-SiC) hydrogen atoms adsorb in ortho and para dimer configurations on the graphene sheet, with no demonstrated change in the graphene-substrate interactions [25,26]. For graphene on metal systems such as graphene on $\operatorname{Ir}(111)$ [gr/Ir(111)], hydrogen adsorption configurations are to some extent guided by graphene-substrate interactions [19,27,28].

The vibrational and configurational changes induced by exposing the $\mathrm{gr} / \mathrm{Ir}(111)$ system to hydrogen atoms are elucidated in this work in terms of hydrogen atom binding sites, influence on the graphene-substrate interaction, and the evolution of the graphene lattice phonons. Through investigation of

\footnotetext{
*linekyhl@inano.au.dk

†liv@phys.au.dk

${ }^{\ddagger}$ regis.bisson@univ-amu.fr
}

high-resolution electron energy loss spectroscopy (HREELS) data, the electronic properties of hydrogenated $\operatorname{gr} / \operatorname{Ir}(111)$ are additionally discussed, providing a solid reference for future chemical functionalization experiments on the system.

The electronic and structural properties of hydrogenated $\operatorname{gr} / \operatorname{Ir}(111)$ have previously been studied using scanning tunneling microscopy (STM) and angle-resolved photoemission spectroscopy (ARPES) [19]. These techniques show nanostructured hydrogenation, with a pattern defined by the moiré structure [29] and the opening of a gap in the band structure at the Dirac point of at least $450 \mathrm{meV}$ [19]. The moire structure arises due to the mismatch between the graphene and the $\operatorname{Ir}(111)$ lattices and gives rise to both a geometric corrugation and an electronic modulation of the density of states on the graphene surface [29-31]. STM images reveal that hydrogen adsorption at low coverage is favored on hcp and/or fcc areas of the moiré pattern, where every second carbon atom is positioned directly above an iridium atom. See Fig. 1(a). Based on these observations and supporting density functional theory (DFT) calculations, Balog et al. [19] proposed that the chemisorption of hydrogen on $\operatorname{gr} / \operatorname{Ir}(111)$ is stabilized by the formation of a graphane chair-like structure [32], where the neighboring carbon atoms within the hexagon are chemically bound to $\mathrm{H}$ above and $\mathrm{Ir}$ below in an alternating way. See Fig. 1(b). This configuration facilitates the rehybridization of carbon from $s p^{2}$ to $s p^{3}$ in a similar manner as observed in diamond and graphane [19].

In contrast, Kim et al. [33] suggested that hydrogenation of $\mathrm{gr} / \operatorname{Ir}(111)$ results in the formation of true graphane islands, where hydrogen atoms are bonded on both sides of the graphene. See Fig. 1(c). This suggestion is based on sum frequency generation (SFG) vibrational spectroscopy measurements, showing a vibrational mode at $353 \mathrm{meV}$. This energy corresponds to the experimentally measured symmetric stretching mode of a purely $s p^{3}$ hybridized $\mathrm{C}-\mathrm{H}$ bond on a hydrogen terminated diamond [C(111)] surface [34-36]. The energy is additionally close to being in the range of energies reported for $\mathrm{C}-\mathrm{H}$ stretches in several alkanes and alkenes on 
(a)

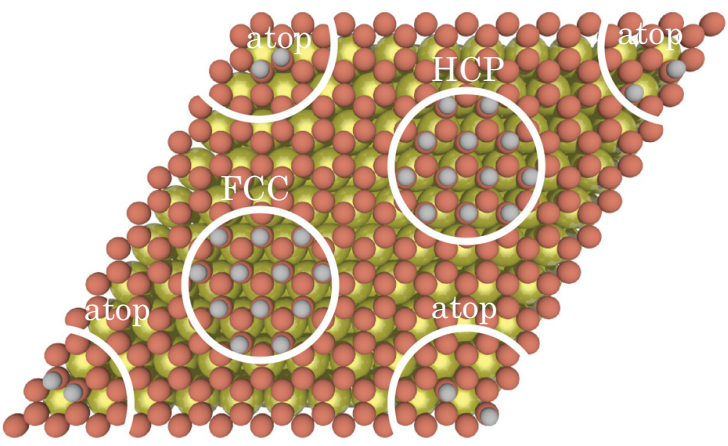

(b)

(c)

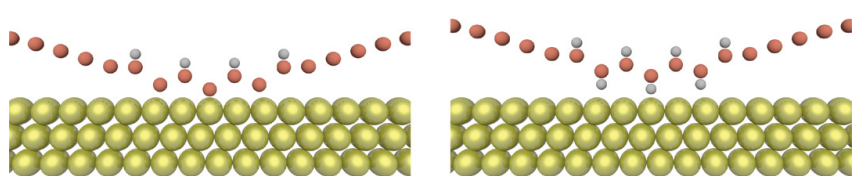

FIG. 1. Schematic drawing of hydrogen exposed graphene on $\operatorname{Ir}(111)$. (a) Hydrogen clusters formed on the fcc and/or hcp sites on the moiré and hydrogen ortho and para dimer structures formed on the more freestanding parts of the moiré (e.g., in the atop sites region). (b) Graphane chair-like structure where hydrogen adsorbs on the top side of the graphene sheet and the underlying Ir atoms act as substitutes for hydrogen adsorbing on the bottom side of the graphene sheet. (c) Graphane chair structure with hydrogen adsorbed on both sides of the graphene sheet. The corrugation is highly exaggerated.

metal surfaces: 355-368 meV [37-41]. In the work of Kim et al. the vibrational signature at $353 \mathrm{meV}$ was only visible when both hydrogen and deuterium atoms were coadsorbed, in agreement with infrared selection rules applied on an ideal graphane layer [33,42].

The formation of true graphane islands would require the presence of $\mathrm{H}(\mathrm{D})$ atoms at the graphene-Ir(111) interface. Indeed, the STM and x-ray photoemission spectroscopy (XPS) measurements with graphene islands on $\operatorname{Ir}(111)$ indicate that hydrogen intercalation can occur in this system [43]. However, it is presently unclear if and how hydrogen intercalation would occur on an $\operatorname{Ir}(111)$ surface fully covered with graphene. Similar studies of graphene on metal substrates indicate that the graphene quality plays a critical role in connection with intercalation $[28,44-50]$. Even in the case where hydrogen is intercalated under $\mathrm{gr} / \mathrm{Ir}(111)$ it is unclear how this would lead to the moiré-related hydrogen pattern observed in STM [19].

In addition to the moiré driven $\mathrm{H}$ structure, a less stable configuration where hydrogen atoms bind as dimers in an ortho or para conformation has been considered [27,51]. These dimers are thought to form in the areas of the moiré unit cell where the positions of the iridium and carbon atoms do not coincide. In these areas the graphene sheet is considered more freestanding and the adsorption scheme of the hydrogen atoms resembles that of graphite [26].

In the present work we study the binding scheme of hydrogen atoms on graphene layers of various quality on an $\operatorname{Ir}(111)$ substrate using HREELS. The HREELS investigation includes the evolution of graphene spectra with increasing hydrogen coverage, with isotopic replacement of hydrogen by deuterium and with thermal annealing. The presented data enable the clarification of vibrational signatures of a somewhat complex spectrum as well as the description of the binding of hydrogen to $\mathrm{gr} / \mathrm{Ir}(111)$ and its effects on the graphene lattice phonons.

\section{EXPERIMENT}

All experiments were performed under ultrahigh-vacuum (UHV) conditions with a base pressure of $2 \times 10^{-10} \mathrm{mbar}$. The experimental setup comprises several interconnected chambers allowing for sample preparation and HREELS measurements without exposing the sample to air. The sample preparation chamber is equipped with an Omicron EFM-H thermal atomic source, through which a beam of hydrogen or deuterium atoms is produced at $2700 \mathrm{~K}$, by molecular dissociation on the walls of a hot tungsten capillary. The atomic hydrogen (deuterium) flux was set to $5 \times 10^{12}$ and $5 \times 10^{13} \mathrm{~cm}^{-2} \mathrm{~s}^{-1}$ for the isotopic substitution and $\mathrm{H}$ saturation deposition, respectively. Vibrational and electronic spectra were obtained using high-resolution electron energy loss spectroscopy (HREELS) with a VSI delta 0.5 spectrometer, where $\Theta_{\mathrm{i}}=$ incidence angle and $\Theta_{\mathrm{s}}=$ scattering angle are defined with respect to the normal to the sample surface. It was found that spectra obtained for $\mathrm{gr} / \mathrm{Ir}(111)$ recorded in the specular direction, $\Theta_{\mathrm{s}}=\Theta_{\mathrm{i}}=62^{\circ}$, display a high background loss due to excitations of electron-hole pairs in addition to an intense plasmonic excitation feature in the area of C-H vibrational losses $(\sim 0.3-0.5 \mathrm{eV})$. However, the plasmon disperses upward in frequency and damps at higher transferred momentum (see Supplemental Material [52] S1). Therefore most measurements were performed out-of-specular $\left(\Theta_{\mathrm{s}} \neq \Theta_{\mathrm{i}}\right)$. Two incident energies were used: 20 and $5 \mathrm{eV}$. These were found to be optimal for measuring, respectively, phonon and plasmon modes and C-H/C-D vibrations. Since the typical momentum transfer for the excitation in the phonon region was less than $0.1 \AA^{-1}$, HREELS measurements presented here probe the phonon spectrum near the $\Gamma$ point of the graphene Brillouin zone. The energy resolution determined by the full width at half maximum (FWHM) of the elastic peak was typically $4-5 \mathrm{meV}$.

$\mathrm{Gr} / \operatorname{Ir}(111)$ samples were either prepared in situ at AixMarseille Université, France, or ex situ at Aarhus University, Denmark, and shipped to Aix-Marseille Université, France. The samples were in both cases prepared by (1) Cleaning the $\operatorname{Ir}(111)$ surface by continuous cycles of $\mathrm{Ar}^{+}$ion sputtering and subsequent annealing to $1173 \mathrm{~K}$. (2) Growing the graphene by running 15 cycles of annealing at $473-1508 \mathrm{~K}$ in an ethylene background pressure of $4 \times 10^{-7}$ mbar.

Graphene samples used throughout the experiments were of two different qualities: (i) High-quality single-domain graphene with low defect density and (ii) low-quality multidomain graphene with high rotational disorder. STM images and low-energy electron diffraction (LEED) patterns allowed for verification of the sample qualities (see Supplemental Material [52] S2). The HREEL spectra of the two sample types showed similar loss features for both clean and hydrogenated $\mathrm{gr} / \mathrm{Ir}(111)$ and were found to differ only with respect to the following: A higher scattering for the low-quality samples was found giving rise to lower counts in the zero-loss peak compared to the high-quality samples (see [52] for 


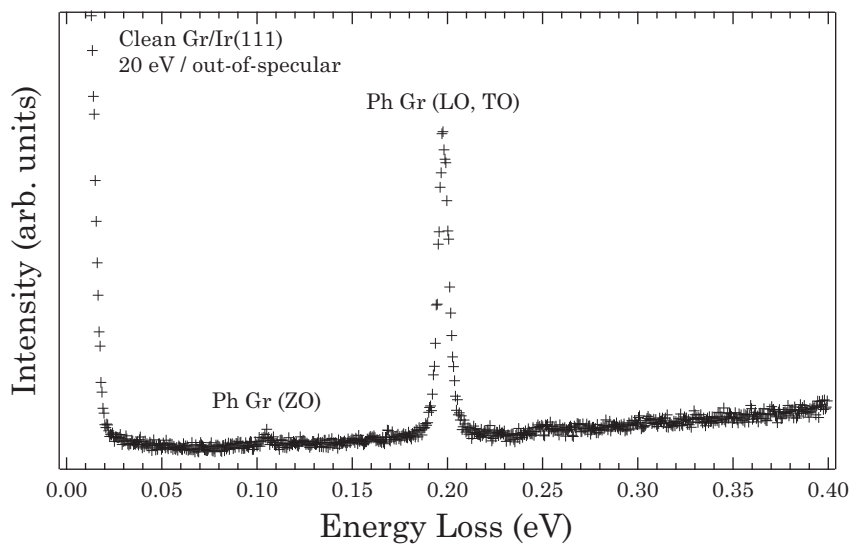

FIG. 2. HREEL spectrum of clean gr/Ir(111). Two energy loss peaks represent, respectively, an out-of-plane optical ( $\mathrm{ZO})$ phonon at $105 \mathrm{meV}$ and two in-plane optical (LO and TO) phonons resolved as one peak at $198 \mathrm{meV}$. The absence of further features in the spectrum indicates the sample is free of any adsorbate contamination.

data analysis details). An additional weak feature related to hydrogen adsorption was found in the spectrum for the low-quality samples. The presented data in Figs. 2, 4(b), and 5 were acquired on high-quality graphene with only one rotational domain $\left(R 0^{\circ}\right)$ whereas the presented data in Figures 3, 4(a), and 6 were obtained from low-quality samples. Data presented in Fig. 7 represent a comparison between a lowand a high-quality graphene exposed to hydrogen.

The samples were annealed to $900 \mathrm{~K}$ between each experiment to desorb hydrogen and retrieve a clean $\operatorname{gr} / \operatorname{Ir}(111)$ surface. The quality and cleanliness of the graphene between experiments was confirmed by LEED and HREELS. The quality was additionally checked by STM immediately after growth and again at the end of the experimental series. It was found that the quality of the graphene did not degrade even after several hydrogen adsorption-desorption cycles.

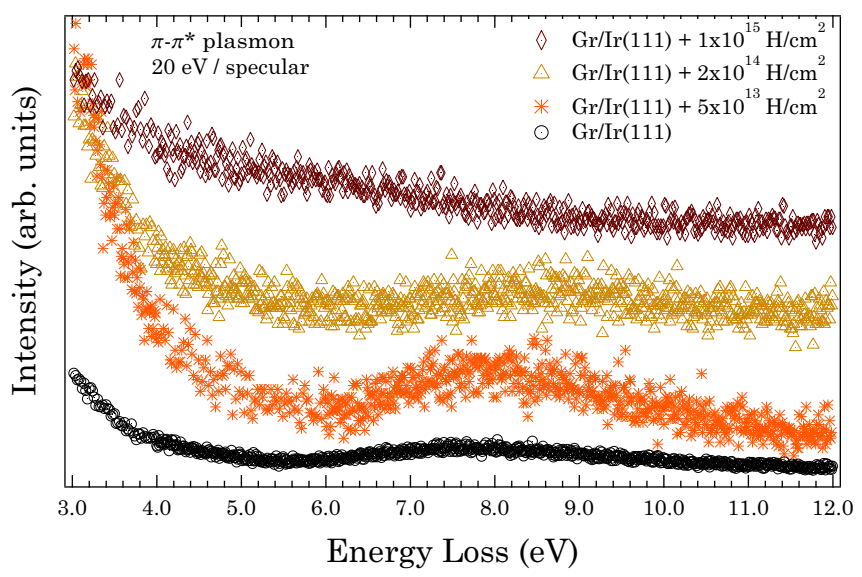

FIG. 3. Spectral evolution of the $\pi-\pi^{*}$ plasmon in $\mathrm{gr} / \mathrm{Ir}(111)$ upon hydrogen atom exposure. The spectra have been offset for clarity. When increasing the hydrogen atom dose the plasmon decreases and finally disappears. The disappearance of the $\pi-\pi^{*}$ plasmon witnesses a loss of metallicity of the graphene due to a disruption of the graphene $\pi$ bands upon increased chemisorbed hydrogen coverage.
Details of the data analysis procedures are given in the Supplemental Material [52].

\section{RESULTS}

Figure 2 displays a HREEL spectrum of clean $\operatorname{gr} / \operatorname{Ir}(111)$ recorded at $20 \mathrm{eV}$ incident energy in out-of-specular geometry. Only two loss features at 105 and $198 \mathrm{meV}$ are present. Based on previous HREELS experiments on clean HOPG [53] these peaks are assigned to graphene sheet phonons: The lowerenergy peak at $105 \mathrm{meV}$ corresponds to an out-of-plane optical (ZO) phonon mode and the higher-energy peak at $198 \mathrm{meV}$ contains two in-plane optical phonon modes [transversal (TO) and longitudinal (LO)], that are very close in energy [54,55]. The absence of any additional peaks in the HREEL spectrum indicates a clean sample with no physisorbed or chemisorbed species.

HREELS studies of plasmon excitations in graphene, in particular the dispersion of the sheet plasmon $(0.5-1.5 \mathrm{eV})$, $\pi-\pi^{*}$ plasmon $(\sim 5-8 \mathrm{eV})$, and $\sigma+\pi$ plasmon $(\sim 15-25 \mathrm{eV})$ have been presented in the literature [56-61]. On a brief side note it must, however, be mentioned that the question of whether these loss features are in fact merely single-particle charge resonances, rather than collective oscillations, is under ongoing discussion [62-64]. In Fig. 3 the evolution of the $\pi-\pi^{*}$ plasmon in $\mathrm{gr} / \mathrm{Ir}(111)$, with increasing levels of hydrogenation, is presented. For clean $\mathrm{gr} / \operatorname{Ir}(111)$, the background is low and the broad plasmonic feature centered around $8 \mathrm{eV}$ is relatively small in intensity. The position of the $\pi-\pi^{*}$ plasmon, in our case, is at higher energy than what has previously been reported for freestanding graphene [59], graphene on $\mathrm{SiC}[25,56]$, graphene on $\mathrm{Pt}$ [58], and graphene on $\mathrm{Ni}(111)$ [60,61] ranging from 4.7 to $7.5 \mathrm{eV}$, at zero momentum transfer. The high energy observed in our work may be related to the polycrystalline graphene (see S2 [52]) used in this experiment. It has been reported that for increased corrugation, such as that of the graphene on $\mathrm{Ru}(0001)$ system, the plasmon becomes localized in a higher-energy state [65]. In our polycrystalline samples the graphene domains may give rise to such localization effects yielding a higher-energy $\pi-\pi^{*}$ plasmon. Performing plasmon dispersion measurements on a polycrystalline $\mathrm{gr} / \mathrm{Ir}(111)$ sample would verify our hypothesis if the dispersion relation is qualitatively similar to that of the graphene on $\mathrm{Ru}(0001)$ system [65]. As the surface is exposed to a relatively small dose of hydrogen atoms, corresponding to $5 \times 10^{13} \mathrm{H} \mathrm{cm}^{-2}$, the background of the spectrum increases rapidly. This happens most likely due to doping and a corresponding change in the density of available charge carriers. Adding more hydrogen atoms to the surface, however, leads to a decrease of the plasmon intensity until its final extinction for a hydrogen exposure above $1 \times 10^{15} \mathrm{H} \mathrm{cm}^{-2}$. This behavior is qualitatively in agreement with angle-resolved photoemission spectroscopy reported for this system, where the graphene $\pi$ bands broaden and are disrupted upon increasing the hydrogen dose [19]: Adding hydrogen atoms to $\operatorname{gr} / \operatorname{Ir}(111)$ was found to open a gap in the band structure at the Dirac point accompanied by a broadening of the bands [19]. With increasing hydrogen dose the $\pi$ bands broaden significantly until their complete disappearance at high $\mathrm{H}$ coverage. This behavior is in agreement 

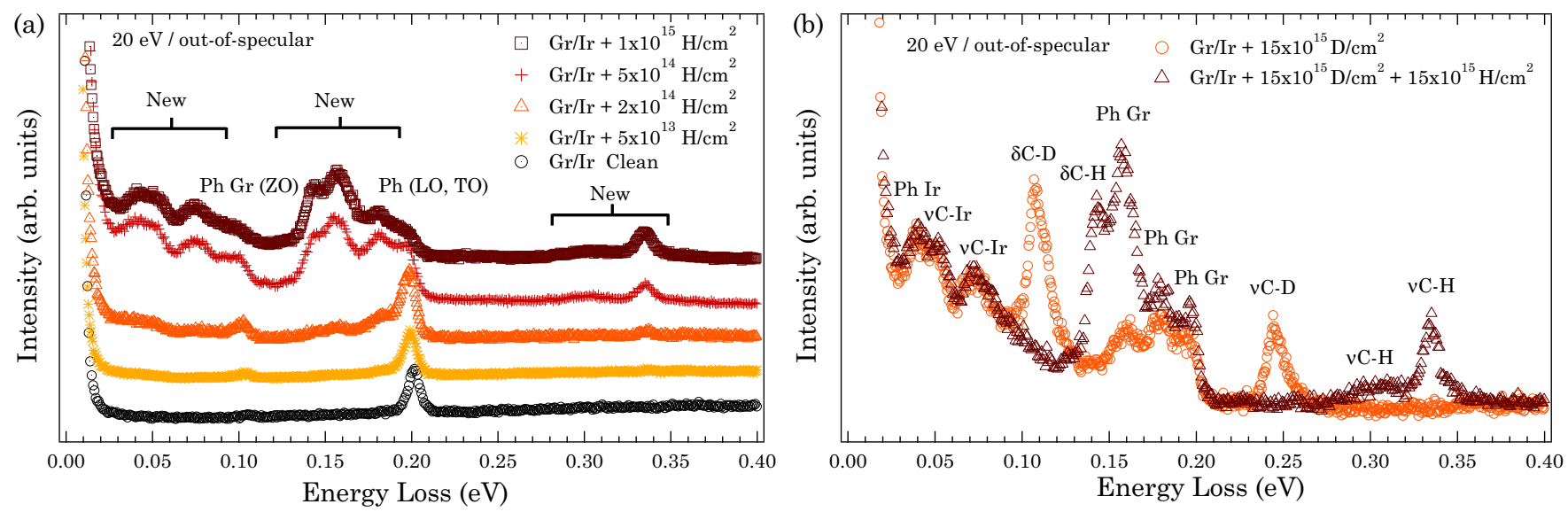

FIG. 4. (a) The evolution of the HREEL spectra of $\mathrm{gr} / \mathrm{Ir}(111)$ upon increasing doses of hydrogen atoms. The ZO and LO/TO phonons of the clean $\mathrm{gr} / \operatorname{Ir}(111)$ remain distinguishable even at saturation coverage. New features arise in the range of 280-360 meV as well as 105-180 and 30-80 meV. All features are discussed and assigned in the text to either phonon modes or adsorbate vibrations. The spectra have been offset for clarity. (b) Isotopic substitution of deuterated gr/Ir(111): HREEL spectrum of gr/Ir(111) exposed to deuterium atoms (circles). Subsequently the same sample was exposed to hydrogen atoms (triangles). The isotopic substitution allows for attribution of peaks common in the two spectra to phonons and H/D-independent vibrations, while peaks changing in frequency are attributed to C-H or C-D related vibrations, respectively, for the two spectra.

with the observed annihilation of the $\pi-\pi^{*}$ plasmon in our experiment.

Removal of conduction electrons by $\mathrm{H}$ adsorption has a profound influence on the transparency of graphene towards the incident electron beam. It has been shown previously that, for hydrogenated quasifreestanding graphene on $\mathrm{H}-\mathrm{SiC}$, an incident electron beam is able to sense the $\mathrm{H}-$ $\mathrm{SiC}$ substrate $[25,66]$. A similar effect is also observed on hydrogenated $\mathrm{gr} / \operatorname{Ir}(111)$, allowing an Ir substrate phonon to be probed (see Sec. IV).

Figure 4(a) demonstrates how components of the graphene sheet phonon associated with hydrogenated areas appear as the hydrogen coverage is increased. Additional C-H stretching and

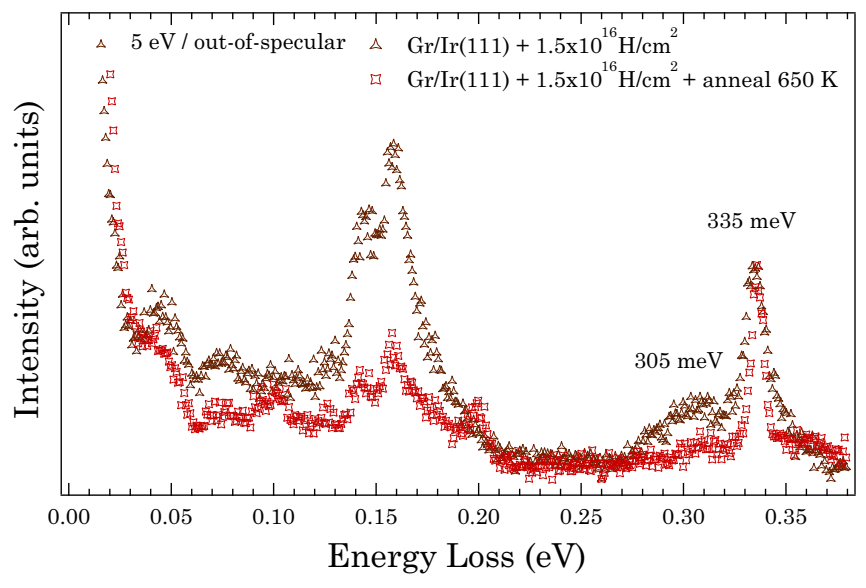

FIG. 5. Spectra of a saturated hydrogenated $\operatorname{gr} / \operatorname{Ir}(111)$ (triangles) and a hydrogenated $\mathrm{gr} / \operatorname{Ir}(111)$ sample annealed to $650 \mathrm{~K}$ after saturation. The broad $\mathrm{C}-\mathrm{H}$ stretch centered at $305 \mathrm{meV}$ decreases and the peak at $335 \mathrm{meV}$ narrows. From this behavior and the literature, the 305- and the 335-meV features are assigned, respectively, to hydrogen dimers and to hydrogen bound in graphanelike cluster configurations on the fcc/hcp sites of the moiré. bending vibrational components appear upon increased hydrogenation yielding a somewhat complex spectrum compared to that of the clean $\mathrm{gr} / \mathrm{Ir}(111)$ sample. The LO/TO and ZO phonon modes for the clean $\mathrm{gr} / \operatorname{Ir}(111)$ are still visible even at saturation coverage, indicating that reaching a level of functionalization involving all $\mathrm{C}$ atoms is not possible. Appearing components related to hydrogen functionalization are marked "New." In order to facilitate the assignment of the numerous spectral features in hydrogenated samples, we followed the change in spectra upon isotopic substitution [67,68]; see Fig. 4(b). Because of the mass difference between hydrogen and deuterium, it is expected that vibrational signatures related to $\mathrm{C}-\mathrm{H}$ bonds will shift to a new frequency smaller by a factor of roughly $1 / \sqrt{ } 2$, while the phonon modes will remain unchanged. A HREEL spectrum obtained after exposing a

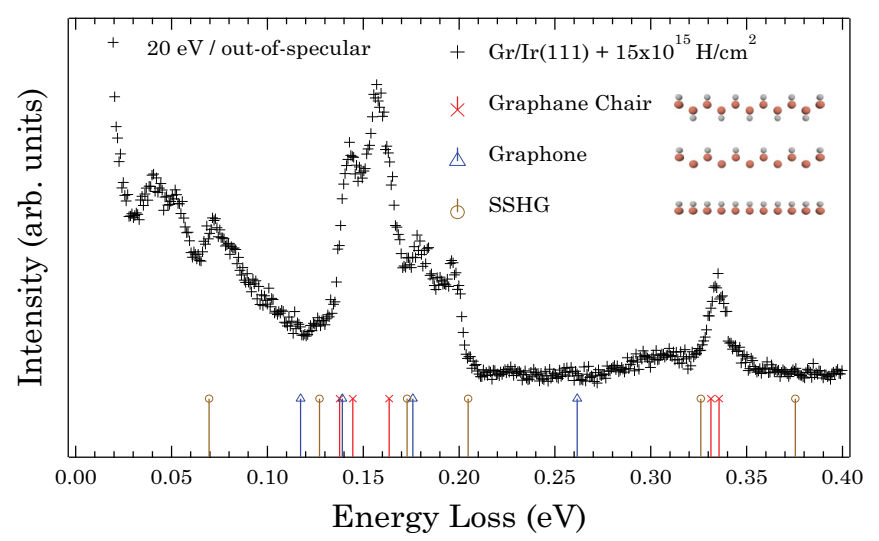

FIG. 6. Comparison of the graphane chair, the graphone, and the SSHG models, including anharmonic corrections, to the experimental HREELS data. Solely the graphane chair model (crosses on bars) reproduces the intense $\mathrm{C}-\mathrm{H}$ stretch peak at $335 \mathrm{meV}$, underpinning the attribution of this vibrational feature to hydrogen bound as graphanelike clusters on the graphene lattice. 


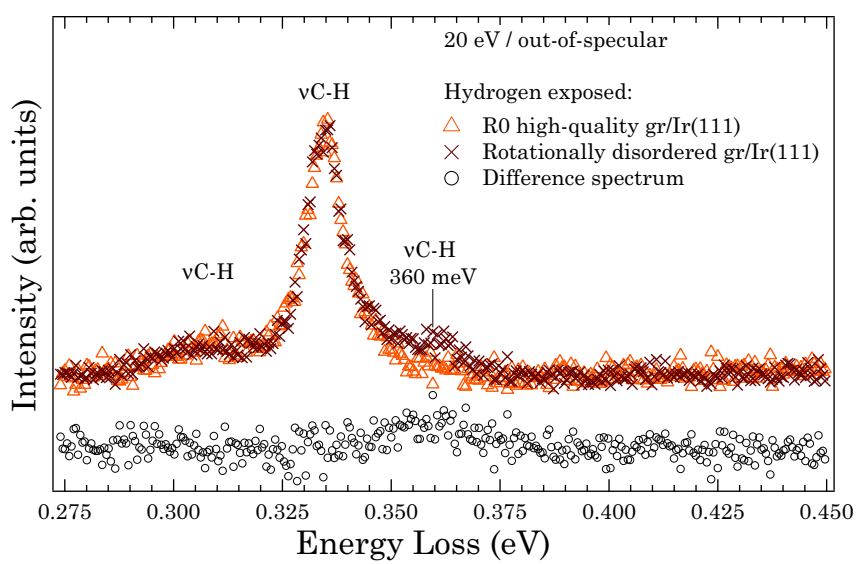

FIG. 7. Comparison of the $\mathrm{C}-\mathrm{H}$ vibrational signatures in the stretching region for low- (crosses) and high- (triangles) quality $\mathrm{gr} / \operatorname{Ir}(111)$ samples. A feature of low intensity is present around $360 \mathrm{meV}$ on the low-quality sample only. The broad nature of this feature is evident from the bump centered around $360 \mathrm{meV}$ appearing in the difference spectrum (circles) shown below the two spectra. This may be assigned to hydrogen adsorbed at edges or domain boundaries of the multidomain low-quality sample.

clean graphene to a $15 \times 10^{15} \mathrm{D} \mathrm{cm}^{-2}$ dose is displayed (circles) in Fig. 4(b). The sample was subsequently exposed to $15 \times 10^{15} \mathrm{H} \mathrm{cm}^{-2}$ to obtain a fully hydrogenated sample by isotopic substitution. The corresponding HREEL spectrum (triangles) is also presented in Fig. 4(b) and shows no difference with a spectrum obtained from a clean $\mathrm{gr} / \operatorname{Ir}(111)$ sample following direct exposure to $\mathrm{H}$ [compare with Fig. 4(a)]. These results indicate a complete replacement of deuterium by hydrogen upon exposure. The spectrum of the deuterated sample [Fig. 4(b), circles] differs from that of the hydrogenated sample (triangles) in two respects: Firstly, the broad feature peaking at $245 \mathrm{meV}$ in the deuterated sample corresponds to the 335-meV peak in the hydrogenated sample. The 335and 245-meV spectral features resemble those observed on hydrogenated (deuterated) gr/H-SiC [25] and hydrogenated (deuterated) HOPG [53] and are therefore readily assigned to $\mathrm{C}-\mathrm{H}$ and C-D stretching vibrations, respectively. Additionally, a bump centered around $305 \mathrm{meV}$ and extending between
280 and $320 \mathrm{meV}$ appears when substituting deuterium by hydrogen; thus we also link this feature to $\mathrm{C}-\mathrm{H}$ vibrations. Secondly, the observed peaks at $108 \mathrm{meV}$ for a deuterated sample and at $143 \mathrm{meV}$ for a hydrogenated one are linked through the $\sim 1 / \sqrt{ } 2$ relationship. Similarly to $\mathrm{gr} / \mathrm{H}-\mathrm{SiC}$ [25] and HOPG [53], these peaks are thereby assigned to C-D and $\mathrm{C}-\mathrm{H}$ bending vibrations, respectively. Several spectral features in Fig. 4(b) are common for both the hydrogenated and deuterated system and they are now briefly discussed. The two peaks at 158 and $178 \mathrm{meV}$ appear gradually with increasing $\mathrm{H}$ dose [Fig. 4(a)] while the 198-meV phonon of clean graphene reduces in intensity and shifts to $\sim 195 \mathrm{meV}$. Therefore, the appearance of these two additional peaks is likely related to the evolution of the sheet phonons of the clean graphene upon hydrogenation. Indeed, the graphene lattice undergoes severe distortion when hydrogen atoms are adsorbed and this will alter the phonon modes in the areas of hydrogenated graphene. The appearance of the additional phonon branches in the vicinity of the 198-meV TO/LO phonon of clean graphene will be discussed in Sec. IV. Similarly, the 105-meV ZO phonon intensity reduces upon hydrogenation and its position shifts to $100 \mathrm{meV}$. Additionally three peaks at 40,52, and $72 \mathrm{meV}$ are common for the deuterated and hydrogenated samples [Fig. 4(b)]. These peaks will be discussed in Sec. IV. The resulting assignment of all vibrations and phonon modes is summarized in Table I.

The C-H stretching vibrational signature comprises two components: A broad feature centered around $305 \mathrm{meV}$ and a peak at $335 \mathrm{meV}$. The latter one has an asymmetric shape with a FWHM of roughly $13 \mathrm{meV}$. This is larger than the typical resolution of $4-5 \mathrm{meV}$ set for our HREELS measurements. The corresponding C-D feature at $255 \mathrm{meV}$ exhibits a similar structure. This indicates that the C-H and C-D vibrational features are each due to contributions from various adsorption configurations with slightly modified vibrations. The spectrum becomes, however, simplified upon annealing. In Fig. 5 two HREEL spectra of $\operatorname{gr} / \operatorname{Ir}(111)$, exposed to a hydrogen dose of $15 \times 10^{15} \mathrm{H} \mathrm{cm}^{-2}$ at room temperature and after a subsequent anneal to $650 \mathrm{~K}$ are displayed. Following the annealing the broad feature around $305 \mathrm{meV}$ strongly decreased and the $335-\mathrm{meV}$ peak narrowed to the instrumental resolution.

TABLE I. Summary of observed vibrational features in the HREEL spectra of clean and hydrogenated (deuterated) gr/Ir(111).

\begin{tabular}{|c|c|c|c|}
\hline Vibrational mode & $\mathrm{H}$ adsorption (meV) & $\mathrm{D}$ adsorption (meV) & Clean gr/Ir(111) (meV) \\
\hline Phonon Ir & 40 & 40 & \\
\hline$\nu \operatorname{Ir}-\mathrm{C}$ & 52 & 52 & \\
\hline$\nu \operatorname{Ir}-\mathrm{C}$ & 72 & 72 & \\
\hline ZO graphene & 100 & 100 & 105 \\
\hline$\delta \mathrm{C}-\mathrm{D}$ & & 108 & \\
\hline$\delta \mathrm{C}-\mathrm{H}$ & 143 & & \\
\hline $\mathrm{TO} / \mathrm{LO}$ graphanelike clusters+dimers & 158 & 158 & \\
\hline $\mathrm{ZO}$ graphanelike clusters & 178 & 178 & \\
\hline TO/LO graphene & 195 & 195 & 198 \\
\hline$v \mathrm{C}-\mathrm{D}$ & & 245 & \\
\hline$v \mathrm{C}-\mathrm{H}$ dimers & $280-320$ & & \\
\hline vC-H graphanelike clusters & 335 & & \\
\hline$v \mathrm{C}-\mathrm{H}$ defects & 360 & & \\
\hline
\end{tabular}




\section{DISCUSSION}

Identification of hydrogen adsorbate structures on gr/Ir(111) have previously been attempted by comparing XPS measurements with calculated values [27]. The experimental spectra can be reproduced by DFT calculations by considering hydrogen chemisorption structures on the graphene top side only (graphane chair-like hydrogen clusters) or, alternatively, by introducing true graphane islands with hydrogen bound to both sides of the graphene. Thus $\mathrm{C} 1 s$ core-level spectroscopy data alone do not allow distinction between graphanelike and true graphane structures. Potentially, a combined XPS and DFT study of the Ir $4 f$ core levels for the two scenarios could provide a distinction. However, at present there are no such studies available in the literature.

The reported XPS data [27] show three C $1 s$ components of different thermal stability related to the hydrogen exposure. The most stable of the three components can be isolated when annealing hydrogenated samples above $630 \mathrm{~K}$. In the present work, a hydrogenated $\mathrm{gr} / \mathrm{Ir}(111)$ sample was similarly annealed to isolate a single $\mathrm{C}-\mathrm{H}$ stretching component at $335 \mathrm{meV}$, which therefore correlates with the hydrogen structures responsible for the $\mathrm{C} 1 s$ signatures at $284.62 \mathrm{eV}$ in Ref. [27]. A definite assignment of this component to a single hydrogen adsorption configuration would require a vibrational study based on DFT of the complex nanostructured hydrogenated $\mathrm{gr} / \mathrm{Ir}(111)$ system. Meanwhile, comparison can be made to DFT based vibrational analyses of simpler systems of hydrogenated graphene: DFT studies have shown that pure graphane can exist in three stable configurations, namely, chair graphane [32,69-72], boat graphane [32,69], and washboard graphane [69]. The calculated phonon spectra at the $\Gamma$ point of these models [69] show that for symmetry reasons, only the chair configuration will present a structured spectrum with well-resolved peaks, as reported here. Therefore, only the graphane chair configuration will be considered in the remainder of the discussion. DFT calculations have also shown that hydrogen addition to graphene leading to graphone [73] or even to fully single-sided hydrogenated graphene (SSHG) [74] is exothermic [71]. The stability of graphone towards other $\mathrm{H}$ configurations has, however, been questioned [75]. These single-sided hydrogenated graphene sheets possess a phonon spectrum at the $\Gamma$ point, which should be structured with well-resolved peaks within the HREELS resolution of the presented experiments. We thus compare (Fig. 6), the DFT calculated vibrational and phonon signatures of the chair graphane, the graphone, and the SSHG models with our HREEL spectra obtained from a hydrogenated sample at saturation coverage. Note that we have modified the theoretical harmonic frequencies according to anharmonic corrections calculated by Sakong and Kratzer for the stretching and bending/phonon modes of a hydrogen monomer [76]. This is justified by the isotopic shift, measured experimentally, of $\sim 1.36$. This is lower than the expected value of $\sim 1.39$ for single-particle harmonic -H/-D oscillators at $300 \mathrm{~K}$ [77]. The value $\sim 1.36$ is, however, obtained if anharmonic effects and vibrations of the nearby carbon atoms in the graphene sheet are included as well $[77,76]$. The calculated values of the vibrational frequencies before and after anharmonic corrections are presented in the Supplemental Material [52] ST1. In Fig. 6 a HREEL spectrum of hydrogen functionalized $\operatorname{gr} / \operatorname{Ir}(111)$ (prepared by isotopic substitution of deuterium) is shown. The theoretical vibrational frequencies of graphane (crosses), graphone (triangles), and SSHG (circles) are indicated with bars. The graphone model cannot reproduce experimental data: The highest $\mathrm{C}-\mathrm{H}$ stretching frequency is found $74 \mathrm{meV}$ lower than the experimentally observed $\mathrm{C}-\mathrm{H}$ stretching frequency. The SSHG model, on the other hand, provides a $\mathrm{C}-\mathrm{H}$ stretching in the middle of the two experimentally obtained $\mathrm{C}-\mathrm{H}$ signatures. However, no experimental signature matches the $\mathrm{C}-\mathrm{H}$ stretching with the highest frequency of the SSHG model. Solely the graphane chair model is able to reproduce the experimentally obtained $\mathrm{C}-\mathrm{H}$ signature at $335 \mathrm{meV}$ and this model will therefore be considered in more detail as follows.

The graphane chair model predicts a group of bend/phonon signatures around 141 and $163 \mathrm{meV}$, which are close to the experimental features measured at 143 and $158 \mathrm{meV}$. By isotopic substitution we have demonstrated that the $143-\mathrm{meV}$ signature is related to a $\mathrm{C}-\mathrm{H}$ bending mode $[25,53,71,78]$. However, in Figs. 4 and 5 it clearly appears that the intensities of both the $143-\mathrm{meV}$ bending and the $158-\mathrm{meV}$ phonon are affected by the hydrogen coverage. This behavior suggests a strong coupling between the $\mathrm{C}-\mathrm{H}$ bending mode and the $158-\mathrm{meV}$ phonon. This experimentally observed coupling is a potential verification of a recent theoretical work by Huang et al. [72] showing that the LO/TO phonon mode of the graphane chair at $163 \mathrm{meV}$ has a strong $\mathrm{C}-\mathrm{H}$ bending character that vanishes when substituting hydrogen by deuterium.

The graphane chair model [72] additionally predicts that a ZO phonon is present around $140 \mathrm{meV}$. No phonon-related feature at this energy was observed in our HREEL spectra, as evidenced in the deuterated sample [Fig. 4(b)]. Instead, vibrational features, not yet assigned, at 40, 52, 72, and $178 \mathrm{meV}$, are observed. In contrast to LO and TO phonons, which are sensitive to the in-plane pattern of hydrogen adsorption [69], the $\mathrm{ZO}$ phonon is sensitive to the carbon lattice distortion perpendicular to the honeycomb sheet. The observed discrepancy for the $\mathrm{ZO}$ phonon between the graphane chair model and the HREELS measurements suggests that the corrugation of the hydrogenated $\operatorname{gr} / \operatorname{Ir}(111)$ is different to that of a pure graphane chair. A comparison of phonon spectra for the graphane chair, graphone, and SSHG models [71] indicates that the position of the $\mathrm{ZO}$ branch at the $\Gamma$ point is strongly affected by the hydrogen configuration and can be shifted above or below the energy of clean $\operatorname{gr} / \operatorname{Ir}(111)$. The position of the $\mathrm{ZO}$ phonon of the hydrogen exposed $\mathrm{gr} / \mathrm{Ir}(111)$ is therefore yet to be identified in experiment.

The first peak at $40 \mathrm{meV}$ can be identified as an Ir lattice vibration on a clean $\operatorname{Ir}(111)$ surface [79]. This phonon energy is in good agreement with the Debye cutoff frequency derived from the Debye temperature for iridium of $430 \mathrm{~K}$ [79]. The fact that a phonon signature of the clean $\operatorname{Ir}(111)$ surface is observed on a hydrogenated $\operatorname{gr} / \operatorname{Ir}(111)$ sample is not surprising. Indeed, we have shown in Fig. 3 that the metallicity of the graphene sheet is lost upon hydrogen adsorption making it transparent to the electron probe beam. Furthermore, XPS measurements [27] have shown that the hydrogen coverage of the graphene layer at saturation is far from being complete, leaving significant parts of the carbon sheet clean. This is in line with the findings of this work where a remaining signature of TO/LO (195 meV) and ZO phonons (100 meV) of clean graphene is present in the HREEL spectra 
even after hydrogen saturation shown in Fig. 4(a). The second two peaks at 52 and $72 \mathrm{meV}$ are in the area of metal-carbon stretching vibrational modes measured for systems like benzene adsorbed on $\mathrm{Rh}(111)$ [80] (rhodium belong to the same group of the Periodic Table as iridium): Two peaks at 43 and $68 \mathrm{meV}$ were assigned to the Rh-C stretching vibrations of the benzene molecule adsorbed flat on the $\mathrm{Rh}(111)$ surface. Given that the desorption temperature/binding energy of benzene on $\operatorname{Rh}(111)$ [80] and $\operatorname{Ir}(111)$ [81] are alike, the metal-carbon stretching vibrations should be similar on both surfaces. We thus tentatively assign the features at 52 and $72 \mathrm{meV}$ to $\mathrm{Ir}-\mathrm{C}$ bonds formed in the hydrogenated $\mathrm{gr} / \operatorname{Ir}(111)$ samples, This leaves the 178-meV spectral feature unassigned. We propose that this signature corresponds to the ZO phonon of graphanelike clusters where carbon atoms are bound alternatingly to hydrogen atoms on the top side and to Ir atoms on the bottom side of the graphene layer.

In Ref. [33] SFG spectroscopy was used to probe hydrogen structures formed on $\mathrm{gr} / \operatorname{Ir}(111)$. Three vibrational peaks related to $\mathrm{C}-\mathrm{H}$ (C-D) modes were found at 318, 337, and $353 \mathrm{meV}(233,251$, and $264 \mathrm{meV})$. The two former vibrations were assigned to para and ortho dimers of hydrogen, and the latter vibration to $s p^{3}$-bound hydrogen in pure graphane chair configurations on the $\operatorname{gr} / \operatorname{Ir}(111)$ sample [33]. In this work the common signature around $335-337 \mathrm{meV}$ has been associated with graphane chair-like clusters, since the graphene chair-like clusters simultaneously account for the two groups of bend/phonon signatures at 143 and $158 \mathrm{meV}$ [72], while the dimer structures do not. The 318-meV signature found in SFG lies in the broad feature we measured between 280 and $320 \mathrm{meV}$. Since SFG obeys several optical selection rules, it is only sensitive to vibrations exhibiting specific symmetries. In contrary, HREELS measurements obtained in out-of-specular geometry rely on an impact mechanism, which does not involve selection rules. Therefore, our spectra are representative of the diversity of hydrogen binding configurations on the sample. Such diversity is not surprising since, e.g., hydrogen dimers will adsorb in slightly different environments depending on their exact position in the freestanding part of the moiré structure. Accordingly, DFT calculations on various dimers [76] and monomers [76,77] yield vibrational signatures that are all at a lower energy than for graphanelike clusters.

Furthermore, in Fig. 5 we have shown that the 280320-meV feature disappears before the 335-meV signature when annealing the hydrogenated $\operatorname{gr} / \operatorname{Ir}(111)$ sample. Such thermal behavior agrees with the XPS data in Ref. [27] where it was shown that the component related to hydrogen dimers has lower stability than the one related to graphanelike clusters. Thus we link the 280-320-meV vibrational feature to dimers, and the $335-\mathrm{meV}$ vibrational peak to graphanelike clusters.

The additional feature at $353 \mathrm{meV}$ observed with SFG [33], related to an $s p^{3} \mathrm{C}-\mathrm{H}$ vibration of hydrogen atoms adsorbed on both sides of the graphene layer was not observed by HREELS on high-quality $\operatorname{gr} / \operatorname{Ir}(111)$ samples. The absence of such signature could be twofold: The impact mechanism cross section may be too low for this specific vibrational mode to be excited, or the density of species related to this signature is negligible in the high-quality samples. The first reason seems unlikely, while the latter cannot be excluded. As a further extension to this line of discussion we note that we have observed a somewhat broad faint loss feature in the energy range 350-370 $\mathrm{meV}$ (Fig. 7) in a rotationally disordered graphene after hydrogen atom exposure. This range of vibrational energies corresponds to certain $s p^{3} \mathrm{C}-\mathrm{H}$ stretching modes found experimentally for hydrogen terminated $\mathrm{C}(111)$ and $\mathrm{C}(100)$ [34,35,82]. Additionally these frequencies match $\mathrm{C}-\mathrm{H}$ stretching modes measured for methane, ethylene, and acetylene adsorbed on transition metals [37-41]. These results indicate that the broad feature in the range 350-370 meV may also originate from the boundaries and edge-site hydrogen in a low-quality sample.

To our knowledge, the only direct proof of hydrogen intercalation of the $\mathrm{gr} / \operatorname{Ir}(111)$ system, reported at present, was performed on graphene islands [43]. Hence, the 353-meV signature may originate from graphene samples of a quality with multiple domains such that hydrogen intercalation can proceed, leading to formation of pure graphane islands. Additionally, as proposed in Ref. [33], lasers utilized for the SFG measurements deteriorate the graphene layer and may be responsible for introducing additional defects contributing to the $s p^{3}$ peak at $\sim 353 \mathrm{meV}$.

\section{CONCLUSION}

Hydrogen atoms were covalently bound to a high-quality graphene sheet on an $\operatorname{Ir}(111)$ surface. The system was studied using HREELS, providing vibrational and electronic information. The change in the electronic properties of the graphene upon hydrogen adsorption was evidenced in the evolution and disruption of the $\pi-\pi^{*}$ plasmon, in accordance with previous work showing the opening of an electronic band gap. The graphene loss of metallicity at hydrogen saturation coverage is reflected by the appearance of an iridium substrate phonon in the HREEL spectrum. Hydrogen atom exposure of $\operatorname{gr} / \operatorname{Ir}(111)$ gave rise to the appearance of additional branches of graphene lattice vibrations. These phonon branches were found to be related to graphanelike surface structures, consistent with several theoretical studies on the graphane chair system. To assign the origin of the numerous vibration spectral features, isotopic abstraction was performed in addition to thermal annealing measurements. By comparing our findings to DFT studies and XPS measurements available in the literature, a consistent picture of the hydrogen adsorption on $\operatorname{gr} / \operatorname{Ir}(111)$ forms: Hydrogen atoms only adsorb on one side of high-quality graphene sheets. Two main hydrogen configurations coexist: Graphanelike clusters in interaction with the Ir substrate and dimers located on the more freestanding graphene parts of the moiré lattice. These two configurations possess different vibrational frequencies and desorption temperatures, and thus different binding energies. An additional hydrogen adsorption configuration with an $s p^{3}$-like vibrational energy was found to exist only on defective rotationally disordered graphene layers. The complete identification of vibrational features of the system with energies up to $400 \mathrm{meV}$ serves as a reference for future vibrational spectroscopy investigations of chemically functionalized graphene on $\operatorname{Ir}(111)$.

\section{ACKNOWLEDGMENTS}

We would like to acknowledge support from the Danish Council for Independent Research and Innovation Fund Denmark (under the National Initiative for Advanced Graphene Coatings and Composites). 
[1] K. S. Novoselov, A. K. Geim, S. V. Morozov, D. Jiang, Y. Zhang, S. V. Dubonos, I. V. Grigorieva, and A. A. Firsov, Science 306, 666 (2004).

[2] S. Reich, J. Maultzsch, C. Thomsen, and P. Ordejon, Phys. Rev. B 66, 035412 (2002).

[3] K. S. Novoselov, A. K. Geim, S. V. Morozov, D. Jiang, M. I. Katsnelson, I. V. Grigorieva, S. V. Dubonos, and A. A. Firsov, Nature 438, 197 (2005).

[4] M. I. Katsnelson, K. S. Novoselov, and A. K. Geim, Nat. Phys. 2, 620 (2006).

[5] K. I. Bolotin, K. J. Sikes, Z. Jiang, M. Klima, G. Fudenberg, J. Hone, P. Kim, and H. L. Stormer, Solid State Commun. 146, 351 (2008)

[6] F. Wang, Y. B. Zhang, C. S. Tian, C. Girit, A. Zettl, M. Crommie, and Y. R. Shen, Science 320, 206 (2008).

[7] F. Bonaccorso, Z. Sun, T. Hasan, and A. C. Ferrari, Nat. Photon. 4, 611 (2010).

[8] S. Chen, L. Brown, M. Levendorf, W. Cai, S.-Y. Ju, J. Edgeworth, X. Li, C. W. Magnuson, A. Velamakanni, R. D. Piner, J. Kang, J. Park, and R. S. Ruoff, ACS Nano 5, 1321 (2011).

[9] D. Prasai, J. C. Tuberquia, R. R. Harl, G. K. Jennings, and K. I. Bolotin, ACS Nano 6, 1102 (2012).

[10] W. B. Hu, C. Peng, W. J. Luo, M. Lv, X. M. Li, D. Li, Q. Huang, and C. H. Fan, ACS Nano 4, 4317 (2010).

[11] R. R. Nair, H. A. Wu, P. N. Jayaram, I. V. Grigorieva, and A. K. Geim, Science 335, 442 (2012).

[12] S. Stankovich, D. A. Dikin, G. H. B. Dommett, K. M. Kohlhaas, E. J. Zimney, E. A. Stach, R. D. Piner, S. T. Nguyen, and R. S. Ruoff, Nature 442, 282 (2006).

[13] H. Sahin and S. Ciraci, Phys. Rev. B 84, 035452 (2011).

[14] T. Kuila, S. Bose, A. K. Mishra, P. Khanra, N. H. Kim, and J. H. Lee, Prog. Mater. Sci. 57, 1061 (2012).

[15] M. Jablan, H. Buljan, and M. Soljacic, Phys. Rev. B 80, 245435 (2009).

[16] K. P. Loh, Q. L. Bao, G. Eda, and M. Chhowalla, Nat. Chem. 2, 1015 (2010).

[17] O. Leenaerts, B. Partoens, and F. M. Peeters, Phys. Rev. B 77, 125416 (2008).

[18] S. Ryu, M. Y. Han, J. Maultzsch, T. F. Heinz, P. Kim, M. L. Steigerwald, and L. E. Brus, Nano Lett. 8, 4597 (2008).

[19] R. Balog, B. Jørgensen, L. Nilsson, M. Andersen, E. Rienks, M. Bianchi, M. Fanetti, E. Lægsgaard, A. Baraldi, S. Lizzit, Z. Sljivancanin, F. Besenbacher, B. Hammer, T. G. Pedersen, P. Hofmann, and L. Hornekær, Nat. Mater. 9, 315 (2010).

[20] T. O. Wehling, A. V. Balatsky, M. I. Katsnelson, A. I. Lichtenstein, K. Scharnberg, and R. Wiesendanger, Phys. Rev. B 75, 125425 (2007).

[21] M. Andersen, L. Hornekaer, and B. Hammer, Phys. Rev. B 86, 085405 (2012).

[22] M. L. Ng, R. Balog, L. Hornekaer, A. B. Preobrajenski, N. A. Vinogradov, N. Martensson, and K. Schulte, J. Phys. Chem. C 114, 18559 (2010).

[23] S. Schumacher, T. O. Wehling, Pr. Lazić, S. Runte, D. F. Förster, C. Busse, M. Petrović, M. Kralj, S. Blügel, N. Atodiresei, V. Caciuc, and T. Michely, Nano Lett. 13, 5013 (2013).

[24] S. Ulstrup, L. Nilsson, J. A. Miwa, R. Balog, M. Bianchi, L. Hornekaer, and P. Hofmann, Phys. Rev. B 88, 125425 (2013).

[25] F. C. Bocquet, R. Bisson, J. M. Themlin, J. M. Layet, and T. Angot, J. Phys. D: Appl. Phys. 47, 094014 (2014).
[26] L. Hornekaer, Z. Sljivancanin, W. Xu, R. Otero, E. Rauls, I. Stensgaard, E. Laegsgaard, B. Hammer, and F. Besenbacher, Phys. Rev. Lett. 96, 156104 (2006).

[27] R. Balog, M. Andersen, B. Jørgensen, Z. Sljivancanin, B. Hammer, A. Baraldi, R. Larciprete, P. Hofmann, L. Hornekær, and S. Lizzit, ACS Nano 7, 3823 (2013).

[28] L. Nilsson, M. Andersen, R. Balog, E. Laegsgaard, P. Hofmann, F. Besenbacher, B. Hammer, I. Stensgaard, and L. Hornekaer, ACS Nano 6, 10258 (2012).

[29] A. T. N'Diaye, J. Coraux, T. N. Plasa, C. Busse, and T. Michely, New J. Phys. 10, 043033 (2008).

[30] J. Coraux, A. T. N'Diaye, C. Busse, and T. Michely, Nano Lett. 8, 565 (2008).

[31] M. Kralj, I. Pletikosić, M. Petrović, P. Pervan, M. Milun, A. T. N'Diaye, C. Busse, T. Michely, J. Fujii, and I. Vobornik, Phys. Rev. B 84, 075427 (2011).

[32] J. O. Sofo, A. S. Chaudhari, and G. D. Barber, Phys Rev. B 75, 153401 (2007).

[33] H. Kim, T. Balgar, and E. Hasselbrink, Chem. Phys. Lett. 546, 12 (2012).

[34] S.-T. Lee and G. Apai, Phys. Rev. B 48, 2684 (1993).

[35] T. Aizawa, T. Ando, M. Kamo, and Y. Sato, Phys. Rev. B 48, 18348 (1993).

[36] R. P. Chin, J. Y. Huang, Y. R. Shen, T. J. Chuang, and H. Seki, Phys. Rev. B 52, 5985 (1995).

[37] J. Yoshinobu, H. Ogasawara, and M. Kawai, Phys. Rev. Lett. 75, 2176 (1995).

[38] T. S. Marinova and D. V. Chakarov, Surf. Sci. 192, 275 (1987).

[39] H. Steininger, H. Ibach, and S. Lehwald, Surf. Sci. 117, 685 (1982).

[40] J. M. Essen, J. Haubrich, C. Becker, and K. Wandelt, Surf. Sci. 601, 3472 (2007).

[41] T. S. Marinova and K. L. Kostov, Surf. Sci. 181, 573 (1987).

[42] T. Balgar, H. Kim, and E. Hasselbrink, J. Phys. Chem. Lett. 4 2094 (2013).

[43] E. Grånës, Ph.D. thesis, Division of Synchrotron Radiation Research, Department of Physics, Lund University, 2014.

[44] L. Kyhl, S. F. Nielsen, A. G. Cabo, A. Cassidy, J. A. Miwa, and L. Hornekaer, Faraday Discuss. 180, 495 (2015).

[45] S. Schumacher et al., Phys. Rev. B 90, 235437 (2014).

[46] S. Schumacher, D. F. Forster, M. Rosner, T. O. Wehling, and T. Michely, Phys. Rev. Lett. 110, 086111 (2013).

[47] M. Petrovic, I. Šrut Rakić, S. Runte, C. Busse, J. T. Sadowski, P. Lazić, I. Pletikosić, Z.-H. Pan, M. Milun, P. Pervan, N. Atodiresei, R. Brako, D. Šokčević, T. Valla, T. Michely, and M. Kralj, Nat. Commun. 4, 2772 (2013).

[48] E. Granas, J. Knudsen, U. A. Schroder, T. Gerber, C. Busse, M. A. Arman, K. Schulte, J. N. Andersen, and T. Michely, ACS Nano 6, 9951 (2012).

[49] R. Larciprete, S. Ulstrup, P. Lacovig, M. Dalmiglio, M. Bianchi, F. Mazzola, L. Hornekær, F. Orlando, A. Baraldi, P. Hofmann, and S. Lizzit, ACS Nano 6, 9551 (2012).

[50] E. Granas, M. Andersen, M. A. Arman, T. Gerber, B. Hammer, J. Schnadt, J. N. Andersen, T. Michely, and J. Knudsen, J. Phys. Chem. C 117, 16438 (2013).

[51] H. Kim, T. Balgar, and E. Hasselbrink, Chem. Phys. Lett. 508, 1 (2011). 
[52] See Supplemental Material at http://link.aps.org/supplemental/ 10.1103/PhysRevB.93.115403 for details of HREELS data analysis, STM and LEED characterisation of gr/Ir(111) samples and theoretical vibrational values for hydrogenated graphene derivatives.

[53] E. Areou, G. Cartry, J. M. Layet, and T. Angot, J Chem. Phys. 134, 014701 (2011).

[54] M. Endlich, A. Molina-Sanchez, L. Wirtz, and J. Kroger, Phys. Rev. B 88, 205403 (2013).

[55] T. Aizawa, R. Souda, S. Otani, Y. Ishizawa, and C. Oshima, Phys. Rev. B 42, 11469 (1990).

[56] J. Lu, K. P. Loh, H. Huang, W. Chen, and A. T. S. Wee, Phys. Rev. B 80, 113410 (2009).

[57] T. Langer, D. F. Forster, C. Busse, T. Michely, H. Pfnur, and C. Tegenkamp, New J. Phys. 13, 053006 (2011).

[58] A. Politano, A. R. Marino, V. Formoso, D. Farias, R. Miranda, and G. Chiarello, Phys. Rev. B 84, 033401 (2011).

[59] T. Eberlein, U. Bangert, R. R. Nair, R. Jones, M. Gass, A. L. Bleloch, K. S. Novoselov, A. Geim, and P. R. Briddon, Phys. Rev. B 77, 233406 (2008).

[60] R. Rosei, S. Modesti, F. Sette, C. Quaresima, A. Savoia, and P. Perfetti, Phys. Rev. B 29, 3416 (1984).

[61] A. V. Generalov and Y. S. Dedkov, Carbon 50, 183 (2012).

[62] F. J. Nelson, J. C. Idrobo, J. D. Fite, Z. L. Miskovic, S. J. Pennycook, S. T. Pantelides, J. U. Lee, and A. C. Diebold, Nano Lett. 14, 3827 (2014).

[63] D. Novko, V. Despoja, and M. Sunjic, Phys. Rev. B 91, 195407 (2015).

[64] T. Stauber, J. Phys.: Condens. Matter 26, 123201 (2014).

[65] A. Politano, D. Campi, V. Formoso, and G. Chiarello, Phys Chem. Chem. Phys. 15, 11356 (2013).

[66] F. C. Bocquet, R. Bisson, J. M. Themlin, J. M. Layet, and T. Angot, Phys. Rev. B 85, 201401 (2012).
[67] T. Zecho, A. Horn, J. Biener, and J. Kuppers, Surf. Sci. 397, 108 (1998).

[68] C. Thomas, T. Angot, and J. M. Layet, Surf. Sci. 602, 2311 (2008).

[69] E. Cadelano, P. L. Palla, S. Giordano, and L. Colombo, Phys. Rev. B 82, 235414 (2010).

[70] H. Peelaers, A. D. Hernandez-Nieves, O. Leenaerts, B. Partoens, and F. M. Peeters, Appl. Phys. Lett. 98, 051914 (2011).

[71] X. H. Zhou, Y. Huang, X. S. Chen, and W. Lu, Solid State Commun. 157, 24 (2013).

[72] L. F. Huang, T. F. Cao, P. L. Gong, and Z. Zeng, Solid State Commun. 190, 5 (2014).

[73] J. Zhou, Q. Wang, Q. Sun, X. S. Chen, Y. Kawazoe, and P. Jena, Nano Lett. 9, 3867 (2009).

[74] B. S. Pujari, S. Gusarov, M. Brett, and A. Kovalenko, Phys. Rev. B 84, 041402 (2011).

[75] Z. Sljivancanin, R. Balog, and L. Hornekaer, Chem. Phys. Lett. 541, 70 (2012).

[76] S. Sakong and P. Kratzer, J. Chem. Phys. 133, 054505 (2010).

[77] C. P. Herrero and R. Ramirez, Phys. Rev. B 79, 115429 (2009).

[78] A. Allouche, Y. Ferro, T. Angot, C. Thomas, and J. M. Layet, J. Chem. Phys. 123, 124701 (2005).

[79] C. J. Hagedorn, M. J. Weiss, and W. H. Weinberg, Phys. Rev. B 60, R14016 (1999).

[80] B. E. Koel, J. E. Crowell, C. M. Mate, and G. A. Somorjai, J. Phys. Chem. 88, 1988 (1984).

[81] C. J. Weststrate, J. W. Bakker, A. C. Gluhoi, W. Ludwig, and B. E. Nieuwenhuys, Surf. Sci. 601, 748 (2007).

[82] B. J. Waclawski, D. T. Pierce, N. Swanson, and R. J. Celotta, J. Vac. Sci. Technol. 21, 368 (1982). 